\title{
Kernos
}

Revue internationale et pluridisciplinaire de religion grecque antique

4 | 1991

Varia

\section{Logos et croyance religieuse chez Héraclite (Fr. 92, 93)}

\author{
Maria E. Koutlouka
}

\section{(2) OpenEdition \\ Journals}

\section{Édition électronique}

URL : http://journals.openedition.org/kernos/306

DOI : 10.4000/kernos.306

ISSN : 2034-7871

\section{Éditeur}

Centre international d'étude de la religion grecque antique

\section{Édition imprimée}

Date de publication : 1 janvier 1991

Pagination : 259-263

ISSN : 0776-3824

\section{Référence électronique}

Maria E. Koutlouka, «Logos et croyance religieuse chez Héraclite (Fr. 92, 93) », Kernos [En ligne],

4 | 1991, mis en ligne le 11 mars 2011, consulté le 03 mai 2019. URL : http://journals.openedition.org/ kernos/306 ; DOI : 10.4000/kernos.306 
Kernos, 4 (1991), p. 259-263.

\title{
LOGOS ET CROYANCE RELIGIEUSE CHEZ HÉRACLITE
}

(Fr. 92, 93)

\author{
À Hélène E. Koutlouka
}

La fin du VIe siècle et les premières décennies du suivant sont, comme on sait, marquées par une renaissance de l'esprit religieux. Héraclite (544-484) cependant pressent le crépuscule de l'ère mythique; influencé par la métaphysique de I'Inde $^{1}$ et par la doctrine zoroastrienne, très au fait de l'orphisme, initié aux mystères d'Éleusis et au culte de Déméter, il est vivement préoccupé par les problèmes religieux de la Grèce. S'il est lui-même animé par une certaine religiosité, il se tient à distance de toutes les traditions. Héritier de la pensée et de la culture helléniques, il se veut le représentant d'une vérité universelle qui soit accessible à tous; il s'exerce ainsi à trouver un équilibre entre la religion et les exigences du Logos.

À Zoroastre il emprunte, dissimulé sous le mythe, le thème de l'éternel conflit entre l'Esprit du mal et l'Esprit Saint; il y décèle la dynamique qui commande la métamorphose des phénomènes physiques.

Adepte de l'initiation et de l'extase, l'orphisme enseigne l'importance de l'élément psychique et conscient pour le salut de l'homme. En dépit de la vénération dont il est l'objet, il est vivement critiqué, condamné même par le philosophe d'Éphèse ${ }^{2}$; les conceptions qu'il véhicule lui semblent encourager une irresponsabilité, une passivité peu compatible avec les idées d'affranchissement de l'homme, de son émancipation de certaines contraintes religieuses et de sa réalisation dans le monde, que l'époque voit se développer.

Héraclite n'éprouve pas davantage d'estime pour les rites appelés mystères, pratiques secrètes qui ont rapport avec la vie future; il les tient

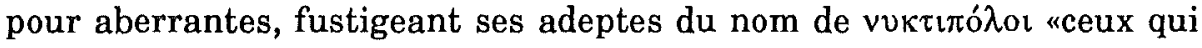
rôdent dans la nuit», en l'occurrence les ténèbres de l'intelligence.

Il est aussi particulièrement acerbe à l'égard du culte orgiastique de Dionysos et il stigmatise les bacchants comme des impies. L'extase dionysiaque détourne l'homme de ses obligations sociales et des problèmes matériels, elle le projette dans la sphère de la subjectivité,
\end{abstract}

1 M.L. WEST, Early Greek Philosophy and the Orient, Oxford, 1971, p. 171 sq.

2 HÉRACLITE, fr. 14a Diels-Kranz. 
l'éloigne de l'univers de la raison et obnubile son esprit. Pareille divagation et dissociation de ce qui fait l'unité psychique de l'homme n'est pas admissible pour le philosophe.

À l'antipode de l'homme éveillé ${ }^{3}$, l'élément mystique se complaît dans le monde inintelligible des rêves qui proposent une vie illusoire, en marge de la vie positive ${ }^{4}$. La conséquence en est un appauvrissement de l'intellect qui perd son aptitude à saisir le sens ${ }^{5}$ et la cohérence du monde ${ }^{6}$, la faculté aussi d'entrer en contact avec l'élément divin ${ }^{7}$. Héraclite est particulièrement formel au sujet de l'existence d'une loi divine, $\theta \varepsilon \hat{\text { ino }}$ vó $\mu \varsigma^{8}$, loi fondatrice qui commande et survit à toutes les lois particulières, loi du Cosmos qui tout régit dans le monde et en assure l'équilibre général.

Dans l'élément mythique présent en toute religion, Héraclite reconnaît un véhicule d'antiques vérités qui remontent à un passé lointain et que le sage ne doit pas rejeter. Mais il condamne comme honteuse, avec une vigueur agressive, la procession phallique de Dionysos et la bacchanale?.

Certains fragments, que nous considérons comme fondamentaux, manifestent la possible concordance de la croyance religieuse avec le Logos. Le fragment 92 évoque la Sibylle, prophétesse d'Apollon, avec des accents si véridiques qu'on croirait qu'Héraclite l'a véritablement rencontrée. Vouée au service du dieu, elle a renié sa nature féminine et délaissé tout enjolivement : elle est sans sourires $(\dot{\alpha} \gamma \varepsilon \dot{\varepsilon} \lambda \alpha \sigma \tau \alpha)$, sans parfums $(\dot{\alpha} \mu v \dot{\rho} \imath \sigma \tau \alpha)$, sans parure $(\dot{\alpha} \kappa \alpha \lambda \lambda \dot{\omega} \pi \imath \sigma \tau \alpha)$, et par sa bouche

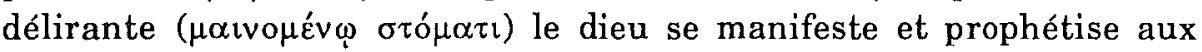
hommes ce qui se produira dans mille ans. Héraclite reconnaît-il vraiment à la Sibylle la capacité de prédire l'avenir lointain ?

Le fragment suivant, le 93, mentionne une autre prophétesse d'Apollon, la Pythie qui, en son nom, rendait les oracles de Delphes. Plutarque, l'auteur le plus qualifié pour parler de cette prêtresse puisqu'il avait fait partie du clergé de Delphes, indique que «la Pythie, élevée dans une maison de pauvres laboureurs, n'apporte au sanctuaire, lorsqu'elle y descend, ni art, ni expérience, ni facultés naturelles;
ID., fr. 89.
ID., fr. 72 .
ID., fr. 30.
ID., fr. I, $2,89$.
ID., fr. 72, 114.
8 ID., fr. 114.
9 ID., fr. 15. 
ignorante à peu près en tout, c'est véritablement une intelligence vierge qui se met en rapport avec Apollon" ${ }^{10}$. Inspirée par le dieu (Ëv $\theta \varepsilon o \varsigma$, $\pi \lambda \dot{p} \rho \eta_{\varsigma} \theta \varepsilon \circ \hat{v}$ ou $\left.\mu \alpha v \varepsilon \hat{\imath} \sigma \alpha\right)$ au moment où elle prononce l'oracle divin, elle était assistée par les prophètes et par un collège de cinq hosioi ${ }^{11}$.

Le trait qui permet de distinguer les pythies des sibylles, c'est que ces dernières étaient douées de la faculté mantique, à la façon d'un privilège personnel, tandis que les premières étaient des organes passifs tout à la disposition du dieu qui les visitait. Bien que l'usage courant confonde souvent $\chi \rho \eta \sigma \mu o ́ \zeta$ et $\mu \alpha v \tau \varepsilon i \alpha$, le sens propre du premier se borne à désigner la réponse oraculaire tandis que le second mot s'applique de façon plus large à la divination en général. Prophétesse du souverain

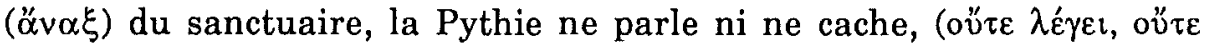
крú $\pi \tau \varepsilon \imath$ ), dit Héraclite, mais donne des signes que les mortels doivent comprendre dans un sens ou dans un autre, l'interprétation étant libre.

L'ambiguïté de ses réponses avait fait qualifier Apollon de Loxias. Sa réponse, en réalité, était claire et formelle quand elle s'adressait à un consultant unique, mais lorsque des tyrans ou des puissantes cités interrogeaient l'oracle sur des affaires importantes, les ministres du dieu, soucieux de leur propre sécurité, évitaient de se compromettre. Un langage équivoque leur était alors d'un précieux secours.

$\mathrm{La}$ question se pose ici de savoir si la formulation claire ou équivoque de l'oracle précédait ou suivait l'énoncé par la Pythie de ses formules souvent versifiées. Ainsi est posé le problème de l'importance reconnue au rôle de la Pythie. Si l'on pense qu'une intervention surnaturelle se produisait au moment de la révélation, on accorde évidemment la primauté à la prêtresse. Mais il est difficile d'admettre qu'une femme illettrée, une simple paysanne, ait pu énoncer des réponses en prose et, à plus forte raison, en hexamètres. Comment ne pas penser que pour une affaire politique d'importance, un traité d'alliance par exemple, la réponse n'ait pas été dictée par la Pythie ? Les questions étant posées par écrit, on peut penser que ce n'est qu'après un certain délai que les réponses élaborées étaient portées à la connaissance des consultants, lesquels devaient attendre ce moment avant d'être admis en présence de la prophétesse.

Mais revenons au philosophe et demandons-nous quelle pensée entendent exprimer les deux fragments que nous venons rapidement de commenter. La foi du monde hellénique en ce dieu Apollon, concerné

10 PluT., Mor., 405c (trad. Bétholaud).

11 Le terme ö $\sigma$ tos s'applique à des personnes consacrées au service divin; la nature de leurs fonctions dans l'acte oraculaire reste inconnue. 
manifestement dans les deux textes, est bien connue. Dieu-Soleil de la lumière qui chasse les ténèbres, dieu de la mesure et de l'équilibre, de l'harmonie et de la sérénité, Phœbus-Apollon est à l'antipode des valeurs que représente Dionysos. Il est naturel dès lors qu'Héraclite ait choisi le premier pour opposer la lumière illusoire d'oracles fondés sur la fiction, qui ne dévoilent rien et ne communiquent que des signes, à la lumière du Logos, la seule qui puisse chasser l'obscurité de l'esprit humain.

Face aux hiérophantes et aux mages - entendons le mot dans son sens péjoratif - qui exercent la mantique trébuchante dans les ténèbres du délire, s'élève la voix stentorienne du philosophe qui, délié de toute servitude religieuse, expose le discours de la raison, de cette vérité unique qu'est le Logos. Si le mage s'efforce de connaître la divinité par ses formes extériorisées ou par l'intermédiaire des différentes formes de divination, l'Homme sage pour sa part arrive à concevoir l'esprit divin par l'entremise du Logos. Aussi tout le cérémonial sacerdotal de la mantique est-il pour lui irrecevable.

Son attitude est la même à l'égard du comportement religieux des hommes en matière de prière et de purification. Il se moque de la vénération accordée aux statues et tourne en ridicule le rituel de purification. Se purifier par le sang, quand on est éclaboussé par le sang, équivaut à vouloir décrotter avec de la boue ; or si quelqu'un se lavait de la boue avec de la boue, il passerait pour un fou à quiconque le verrait ${ }^{12}$. Ainsi pourtant agissent les fidèles. Quand le parricide d'Oreste, souillé du sang de sa mère, implore Apollon de le purifier, l'Oracle lui enjoint de le faire par le sang d'un jeune porc ${ }^{13}$. À Éleusis aussi, le sang de porcs mystiques était agent de purification. Quand Héraclite évoque les porcs se délectant dans la fange plutôt que dans l'eau pure ${ }^{14}$, il se réfêre au $\beta o ́ p ß o p o \varsigma$ des êtres non purifiés des mystères orphiques. Est-ce en les plaçant dans la fange où ils se délectent comme des porcs que l'on peut espérer purifier les impurs ? L'absurdité est manifeste.

Et, pour en revenir au fragment 5 , que penser de ceux qui adressent leurs prières à des statues ? Qui adorent-ils en vérité ? C'est comme s'ils adressaient la parole non aux habitants des maisons mais à des maisons vides. Ils ne connaissent rien de ce que sont les dieux ni les héros.

12 HÉrachite, fr. 5.

13 Cf. EsCH., Eum., 281-283.

14 HÉraClite, fr. 13. 
Avec une grande audace intellectuelle, le philosophe rejette donc ce qu'il considère comme les aberrations religieuses. Ce n'est pas ainsi que l'on peut espérer plaire à la divinité, laquelle ne se conçoit qu'en conformité avec le Logos.

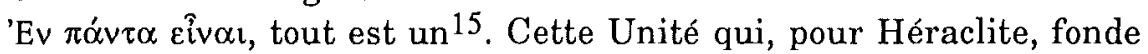
la divinité, comporte une double référence philosophique, au Logos, d'une part, à la Physis et au Cosmos, d'autre part. L'appel à ces derniers termes répond à une volonté de récuser la souveraineté de vieilles figures divines comme Rhéa, Cybèle, Hécate, Mère, et de refuser tous les mythes qui s'y enchaînent. Ce monde, qui est le même pour tous, n'a été créé par aucun dieu ni homme ${ }^{16}$. Et la loi fondamentale de ce monde qui domine toutes les autres lois et qui leur survit, c'est la loi divine que saisit l'intelligence ${ }^{17}$.

Pour Héraclite, la divinité n'a nul besoin des prières, des sacrifices, des offrandes et de tout l'ensemble du rituel auquel recourt la religion populaire pour tenter de se rendre les dieux propices. En réfutant ces croyances et en refusant tout médiateur entre le divin et l'humain, il ne sape pas les bases de la théologie, il ne supprime pas le divin, mais il pense et parle selon l'unique vérité qu'il reconnaît, celle du Logos, qui est le discours éternellement vrai du Cosmos.

Av. Vass. Olgas, 8

Maria E. KOUTLOUKA

GR - 54640 THESSALONIKI

15 ID., fr. 50.

16 ID., fr. 30.

17 ID., fr. 114. 